\title{
Leriche syndrome
}

\author{
Wei-Jing Lee $\cdot$ Yung-Ze Cheng $\cdot$ Hung-Jung Lin
}

Received: 18 January 2008 / Accepted: 20 May 2008 / Published online: 22 August 2008

(C) Springer-Verlag London Ltd 2008

A 45-year-old man with a past history of diabetes mellitus, coronary artery disease with two-vessel disease, and peripheral arterial occlusive disease with gangrene of the left big toe, status post amputation, presented to the emergency department. He was a heavy smoker and complained of severe distension, vomiting, and diffuse abdominal pain for $4-5$ days. Computed tomography (CT) was performed to determine if the pain and distension were caused by intestinal obstruction or ischemic bowel syndrome (Fig. 1). CT scan revealed aortoiliac occlusive disease (Leriche syndrome). The patient underwent bilateral axillofemoral bypass.

Leriche syndrome, also referred to as aortoiliac occlusive disease, is due to thrombotic occlusion of the abdominal aorta just above the site of its bifurcation.

The characteristic symptoms include inability to maintain penile erection, fatigue of both lower limbs, intermittent bilateral claudication with ischemic pain, and absent or diminished femoral pulses along with pallor or coldness of both lower extremities.

W.-J. Lee $\cdot$ Y.-Z. Cheng $(\bowtie) \cdot$ H.-J. Lin

Department of Emergency Medicine, Chi-Mei Medical Center, 901 Chung-Hua Road,

Yung-Kang City, Tainan 710, Taiwan

e-mail: saab931103@yahoo.com.tw
Aortoiliac occlusive disease was first described in 1814 by Robert Graham of London. Leriche syndrome was named after René Leriche, a famous French surgeon who successfully operated "his" syndrome on a 29 -year-old truck driver.

Traditional surgical treatments for aortoiliac occlusive disease are aortoiliac endarterectomy (TEA) and aortobifemoral bypass (AFB). For higher-risk patients, there are alternative ways to avoid abdominal surgery such as axillofemoral bypass (extra-anatomic technique) and percutaneous transluminal angioplasty (PTA) and stenting.

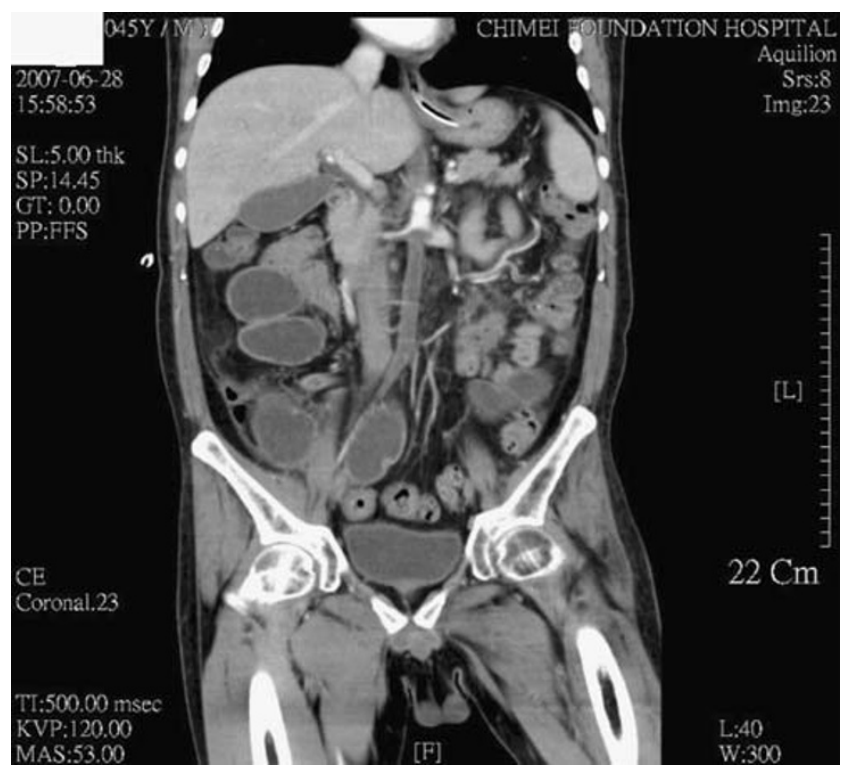

Fig. 1 Coronal reconstruction of contrast-enhanced CT scan of the abdomen revealed thrombosis from the distal abdominal aorta to the bilateral common femoral arteries 